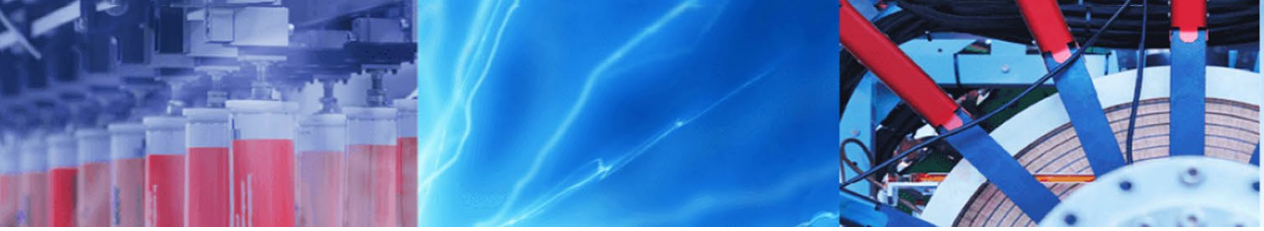

Review Paper

\title{
Investigating the impact of the velocity of a vehicle with a nonlinear suspension system on the dynamic behavior of a Bernoulli-Euler bridge
}

\author{
Babak Shafiei ${ }^{1}$ (1)
}

Received: 18 September 2020 / Accepted: 27 January 2021 / Published online: 8 February 2021

(c) The Author(s) 2021 OPEN

\begin{abstract}
Several authors, utilizing both experimental tests and complicated numerical models, have investigated vehicle speed's impact on a highway bridge's dynamic amplification. Although these tests and models provide reliable quantitative data on frequency contents of the interaction between the two subsystems, engineers should pay further notice to the effects of a subsystem's velocity and the type of suspension system of a vehicle moving over a structure. Hence, in this paper, the dynamic response of a bridge to a moving vehicle is considered. The car is assumed as a quarter car model with both linear and nonlinear stiffness and damping constants. Further, using the modal superposition method, a closed-form solution is obtained for the bridge's vertical response. The results obtained via numerical calculation show a significant increase in the bridge midpoint and total deflection, velocity, and acceleration by increasing the vehicle velocity. Moreover, by neglecting the nonlinear stiffness and damping coefficients of the vehicle suspension system, the bridge's dynamic response remains almost the same with respect to the numerical data. As a general conclusion, it can be claimed that the only significant parameters which can change the dynamic behavior of a bridge subjected to a moving vehicle are the speed of the car and its linear stiffness and damping constants inside its suspension system.
\end{abstract}

Keywords Dynamic response · Bridge · Moving vehicle · Superposition method

\section{Introduction}

Investigating the behavior of bridges under the influence of moving loads has been one of the challenges facing engineering structures over the past few decades. On the one hand, the advancing speed and mass of trains and vehicles, and on the other hand, the request to build lighter structures, make it impossible to overlook the errors caused by the use of the moving load model in the design of bridge structures. The heed given to the topic mentioned above dates back to Willis's efforts [1] and Stokes [2] in the middle of 19 century. For many years it has been commented that when a bridge is subjected to moving loads, the caused dynamic deflections and stresses can be remarkably higher than those seen for the static state [3]. For this reason, numerous works have been devoted to investigating the bridge displacement response using the moving mass [4-8], moving load [9-11], and moving spring-mass models $[12,13]$ for the vehicles.

The moving load can be a good alternative for a vehicle in the displacement studies of the bridge. With this model, it is possible to obtain the bridge's fundamental dynamic characteristics caused by moving cars with a sufficient degree of accuracy. Still, it undergoes shortcoming that the interaction within the bridge and the moving vehicle is neglected. Notwithstanding this drawback, the moving load rule is appropriate for the matter where the automobile's mass is small compared to that of the

Babak Shafiei, babakshafiyi.1994@yahoo.com | 'Department of Civil, Chemical, Environmental, and Materials Engineering - DICAM, University of Bologna, Via Zamboni, 33 Bologna, 40126 Bologna, Italy. 
bridge, solely when the vehicle response is not desired. The moving mass model holds an advantage over the moving load model in that the effect of the inertia of the vehicle is brought into account. Despite that, this model does not reflect the suspension action of the car relative to the bridge. Consequently, to account for the bouncing or suspension movement of the vehicle, the mass-sprung model is granted the most reliable choice to consider in scrutinizing a bridge's dynamic behavior.

A supported beam subjected to a moving sprung mass is adopted to highlight the moving vehicle's impact over the bridge's dynamic behavior, as shown in Fig. 1. The 2-DOF quarter car model with a passive suspension system is assumed as a moving load on the bridge, which is assumed as a Bernoulli-Euler beam resting on two simple supports. Through the modal superposition method, mutually by convolution integrals, closed-form solutions are collected for both the moving car and the bridge, close to the knowledge that repetitions are not taken to update the force applied among the two subsystems to study the typical interaction. For better investigating the impact of vehicle velocity on the bridge, three different constant vehicle speeds are considered in the equation of motion of moving vehicle and bridge. The solution for both vehicle and bridge equations of motion is carried out using Rung-Kutta numerical method (ode45) via MATLAB software. It is considered zero initial conditions of both ends of the bridge mounted on supports and the vehicle at its starting point. Since the 2-DOF quarter car model with a passive suspension system is considered with cubic nonlinearities in this study, the numerical solution can prove whether the nonlinear constants in the passive suspension system can have any potential effect on the dynamic behavior of the bridge. Moreover, the bridge's dynamic increment is obtained via the maximum deflection of the bridge and its peak value of static displacement.

Although many works have been carried out to analyze the dynamic interaction, especially the frequency response [12-14], engineers and researchers should notice the effect of high vehicle speed on the bridge deflection. To substantiate this issue, José [15] investigated and concluded that the maximum dynamic increment factor for a bridge subjected to a highspeed train appears at a critical speed. According to José's research, the displacement and the torsional rotation of the bridge deck at the center of span 11 of Arroyo de las Piedras viaduct in Córdoba-Málaga $\mathrm{HS}$ line were obtained using the Rayleigh method by considering critical train speed of $350 \mathrm{~km} / \mathrm{h}$. Additionally, few tests were carried out to study the dynamic impact factor (DIF) of a bridge subjected to a moving vehicle in recent years. For instance, to extract the DIF of a bridge which is located somewhere in the USA, a field test was conducted by Deng [16] at Lowa State University and bridge engineering center (BEC). The study aimed at the DIF of the bridge subjected to three different trucks and concluded that the DIF increased by applying higher speed to the trucks. However, the mentioned works are solely for transporters with a linear suspension system. Moreover, for many recent theoretical works, a vehicle's suspension system has been considered to have a linear functioning spring or damper, for instance, those listed in Refs. $[17,18]$.

One exception to this research is to study the effect of vehicle speed on a bridge where the vehicle's suspension system is to be assumed to have both linear and nonlinear stiffness and damping constants. This paper aims directly to use the Runge-Kutta method (ode45) since it is widely being used to obtain a highly accurate approximation to the equation of motion, while in the other numerical

Fig.1 Vehicle-bridge model

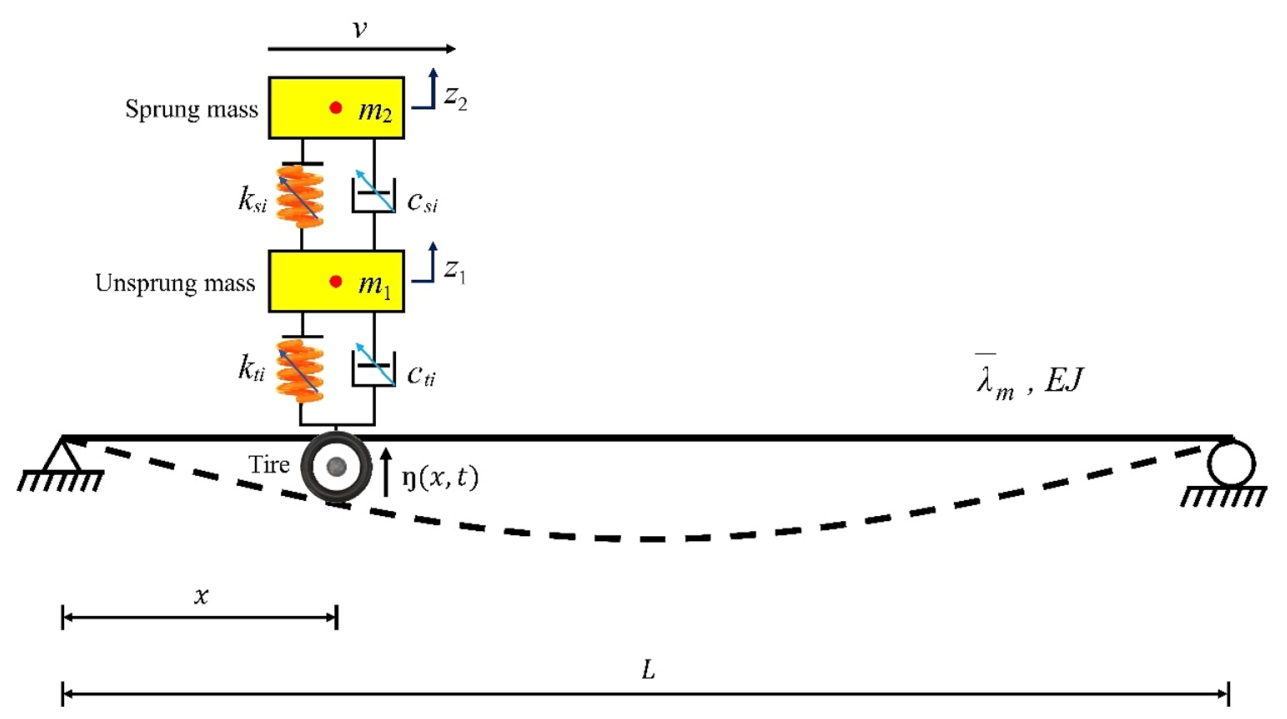

SN Applied Sciences 
methods such as Rayleigh and Newmark-beta, the inaccuracy and uncertainty will occur. For instance, in the Rayleigh method, inaccuracy will rise when the dynamic system is nonlinear, which will cause the damping forces to have an unrealistically large value compared to the restoring forces. Also, in the very recent work done by Nunia and her colleagues [19], the Newmark-beta method is used to solve the coupled dynamic equation of motion of the bride-vehicle. However, the Newmark-beta method is suitable for linear elastic systems, and its performance in nonlinear systems is still not clear. To this end, a 2-DOF quarter car model with a cubic nonlinear passive suspension system is considered, which is moving over a bridge. The bridge is assumed as the Bernoulli-Euler type, and a closed-form solution is obtained using together superposition method, convolution integrals and ode 45 numerical method. The author suggests that further practical investigation be carried out to study the errors obtained from theoretical results and those of field tests.

\section{Assumptions and explanations of the linked vehicle-bridge dynamic model}

The 2-DOF quarter car model with a passive suspension system is considered with cubic nonlinearities moving with constant speed over the bridge, as shown in Fig. 1. The quarter vehicle model is the most generally adopted in the passive suspension systems [20]. In this model, the structural dynamic response depends on the vehicle's movement, expressed as the bridge's total displacement response, which is a two-variable function.

The bridge itself is assumed as a Bernoulli-Euler beam resting on two simple supports, as seen in Fig. 1. This assumption helps to simplify the linear hypothesis of elasticity, which gives a method of measuring the deflection and load-carrying features of the beam. Notice that the damping property or surface distortion of the beam is ignored. The properties of the bridge and vehicle are listed in Table 1 as below $[14,21]$

\section{Equations of motion}

It is vital to understand the system's governing dynamic equations to study the dynamic interaction between a moving vehicle and the bridge. As previously mentioned, the vehicle suspension is presented as a 2-DOF model with cubic nonlinearities. Hence, there are two governing equations for both the car body and unsprung masses. After many manipulations and simplifications, the equation of motion to the vehicle is obtained as:
Table 1 Properties of bridge and vehicle

\begin{tabular}{llll}
\hline Coefficient & Definition & Value & $\mathrm{Unit}$ \\
\hline Vehicle & & & \\
$m_{1}$ & unsprung mass & 364 & $\mathrm{~kg}$ \\
$m_{2}$ & sprung mass & 1776 & $\mathrm{~kg}$ \\
$k_{s 1}$ & spring linear stiffness & $2.8 \times 10^{6}$ & $\mathrm{~N} / \mathrm{m}$ \\
$k_{s 2}$ & spring nonlinear stiffness & $3.0 \times 10^{5}$ & $\mathrm{~N} / \mathrm{m}^{3}$ \\
$k_{t 1}$ & tire linear stiffness & $3.7 \times 10^{7}$ & $\mathrm{~N} / \mathrm{m}$ \\
$k_{t 2}$ & tire nonlinear stiffness & $4.0 \times 10^{6}$ & $\mathrm{~N} / \mathrm{m}^{3}$ \\
$c_{s 1}$ & spring linear damping & $4.0 \times 10^{4}$ & $\mathrm{~N} / \mathrm{m}$ \\
$c_{s 2}$ & spring nonlinear damping & $5.0 \times 10^{4}$ & $\mathrm{~N} / \mathrm{m}^{3}$ \\
$c_{t 1}$ & tire linear damping & $2.0 \times 10^{4}$ & $\mathrm{~N} / \mathrm{m}$ \\
$c_{t 2}$ & tire nonlinear damping & $1.0 \times 10^{4}$ & $\mathrm{~N} / \mathrm{m}^{3}$ \\
$z_{1}$ & unsprung mass displacement & & $\mathrm{m}$ \\
$z_{2}$ & sprung mass displacement & & $\mathrm{m}$ \\
$v$ & vehicle speed & & $\mathrm{m} / \mathrm{s}$ \\
Bridge & & & \\
$L$ & length of the bridge & 15 & $\mathrm{~m}$ \\
$\bar{\lambda} \lambda_{m}$ & total mass per unit length & 28,125 & $\mathrm{~kg} / \mathrm{m}$ \\
$E$ & elastic modulus & $3.5 \times 10^{10}$ & $\mathrm{~N} / \mathrm{m}$ \\
$J$ & moment of inertia & 0.5273 & $\mathrm{~m} 4$ \\
\hline & & &
\end{tabular}

$$
\begin{aligned}
m_{1} \ddot{z}_{1}=- & c_{t 1}\left(\dot{z}_{1}-\frac{\partial \eta(x, t)}{\partial t}\right)-c_{t 2}\left(\dot{z}_{1}-\frac{\partial \eta(x, t)}{\partial t}\right)^{3}-k_{t 1}\left(z_{1}-\eta(x, t)\right) \\
& -k_{t 2}\left(z_{1}-\eta(x, t)\right)^{3}+c_{s 1}\left(\dot{z}_{2}-\dot{z}_{1}\right)+c_{s 2}\left(\dot{z}_{2}-\dot{z}_{1}\right)^{3}+k_{s 1}\left(z_{2}-z_{1}\right) \\
& +k_{s 2}\left(z_{2}-z_{1}\right)^{3}
\end{aligned}
$$

$m_{2} \ddot{z}_{2}=-c_{s 1}\left(\dot{z}_{2}-\dot{z}_{1}\right)-c_{s 2}\left(\dot{z}_{2}-\dot{z}_{1}\right)^{3}-k_{s 1}\left(z_{2}-z_{1}\right)-k_{s 2}\left(z_{2}-z_{1}\right)^{3}$,

which includes all the linearities and nonlinearities for the damping and stiffnesses of the vehicle suspension model moving over the bridge. Further, the equation of motion of the bridge is presented below [22]:

$\bar{\lambda}_{m} \ddot{\eta}+E J \eta^{\prime \prime \prime \prime}=p_{c}(t) \Delta(x-v t)$,

where $x$ and $t$ are contact point coordinate and time, respectively, $\Delta(x-v t)$ denotes the Dirac delta function assessed at the contact point for the vehicle, and $p_{c}(t)$ is the contact force which is equal to:

$p_{c}(t)=-\left(m_{1}+m_{2}\right) g+m_{1} \ddot{z}_{1}+m_{2} \ddot{z}_{2}$,

where $g$ is the gravitational acceleration of Earth's surface.

The solution to Eq. (3) can be expressed in terms of the modal shapes, $\psi_{n}(x)$, and modal coordinates, $r_{b n}(t)$, as:

$\eta(x, t)=\sum_{n} \psi_{n}(x) r_{b n}(t)$. 
The mode shapes of the simply supported beam, which are well investigated by Bao [23], are commonly the sinusoidal type. Hence, Eq. (5) becomes:
Further, through dividing the numerator by denominator of the fraction in Eq. (11), one becomes:

$\ddot{r}_{b n}+f_{b n}^{2} r_{b n}-\frac{2 m_{1}}{\overline{\lambda_{m} L}} \sin \left(\frac{n \pi v t}{L}\right) \ddot{z}_{1}-\frac{2 m_{2}}{\bar{\lambda}_{m} L} \sin \left(\frac{n \pi v t}{L}\right) \ddot{z}_{2}=-\frac{2 m_{1} g}{\bar{\lambda}_{m} L} \sin \left(\frac{n \pi v t}{L}\right)-\frac{2 m_{2} g}{\bar{\lambda}_{m} L} \sin \left(\frac{n \pi v t}{L}\right)$.

$\eta(x, t)=\sum_{n}\left[\sin \left(\frac{n \pi x}{L}\right) r_{b n}(t)\right]$,

where $n$ represents the number of modes. Hence, by substituting Eq. (6) into Eq. (1), the new equation for the vehicle sprung mass becomes:
Finally, three equations, which are Eq. (2), Eq, (7), and Eq. (12), are the overall motion equations which are governing the dynamic system in this study.

$$
\begin{aligned}
m_{1} \ddot{z}_{1}= & -c_{t 1}\left(\dot{z}_{1}-\sum_{n}\left[\sin \left(\frac{n \pi x}{L}\right) \dot{r}_{b n}\right]\right)-c_{t 2}\left(\dot{z}_{1}-\sum_{n}\left[\sin \left(\frac{n \pi x}{L}\right) \dot{r}_{b n}\right]\right)^{3} \\
& -k_{t 1}\left(z_{1}-\sum_{n}\left[\sin \left(\frac{n \pi x}{L}\right) r_{b n}(t)\right]\right)-k_{t 2}\left(z_{1}-\sum_{n}\left[\sin \left(\frac{n \pi x}{L}\right) r_{b n}(t)\right]\right)^{3} \\
& +c_{s 1}\left(\dot{z}_{2}-\dot{z}_{1}\right)+c_{s 2}\left(\dot{z}_{2}-\dot{z}_{1}\right)^{3}+k_{s 1}\left(z_{2}-z_{1}\right)+k_{s 2}\left(z_{2}-z_{1}\right)^{3}
\end{aligned}
$$

where $x=v t$.

In the following, by substituting Eq. (6) into Eq. (3), multiplying both sides of the equation by, and integrating with respect to $x$, Eq. (3) becomes:

\section{Bridge dynamic responses}

In this section, the dynamic responses of bridge and vehicle with three different constant speeds are obtained

$\left.\int_{0}^{L} \bar{\lambda}_{m} \sin \left(\frac{n \pi x}{L}\right) \sum_{n}\left(\sin \left(\frac{n \pi x}{L}\right) \ddot{r}_{b n}\right) d x+\int_{0}^{L} E J \sin \left(\frac{n \pi x}{L}\right) \sum_{n}\left(\sin \left(\frac{n \pi x}{L}\right)\right)^{\prime \prime \prime \prime} r_{b n}\right) d x=\int_{0}^{L} p_{c}(t) \Delta(x-v t) \sin \left(\frac{n \pi x}{L}\right) d x$.

Utilizing the orthogonality conditions for the modal shapes, Eq. (8) becomes:

$\ddot{r}_{b n}+f_{b n}^{2} r_{b n}=\frac{p_{c}(t) \int_{0}^{L} \Delta(x-v t) \sin \left(\frac{n \pi x}{L}\right) d x}{\bar{\lambda}_{m} \int_{0}^{L}\left(\sin \left(\frac{n \pi x}{L}\right)\right)^{2} d x}$,

where $f_{b n}$ represents the frequency of vibration, as:

$f_{b n}=\frac{n^{2} \pi^{2}}{L^{2}} \sqrt{\frac{E J}{\lambda_{m}}}$

Through the calculation of integrals located on the right-hand side of Eq. (9), one becomes: and analyzed to compare the effects of nonlinearities in the quarter 2-DOF vehicle suspension system with linear models studied by others. Using the Rung-Kutta numerical method (ode45), and with the assumption of zero initial conditions for the dynamic system, solution to Eq. (2), Eq. (7), and Eq. (12) are obtained by reducing the orders of differential equations to first orders and forming a system of first-order nonlinear differential equations. Today, it is concluded that good accuracy is received for the beam if we consider only the 1st mode of vibration mentioned by Biggs [24]. Hence, the calculations are solved, considering the first mode of vibration.

$\ddot{r}_{b n}+f_{b n}^{2} r_{b n}=\frac{(2 H(L)-1) H(v t-L H(-L)) H(L H(L)-v t) \sin \left(\frac{n \pi v t}{L}\right)\left(-\left(m_{1}+m_{2}\right) g+m_{1} \ddot{z}_{1}+m_{2} \ddot{z}_{2}\right)}{\frac{1}{4} \bar{\lambda}_{m} L\left(2-\frac{\sin (2 n \pi)}{n \pi}\right)}$. 


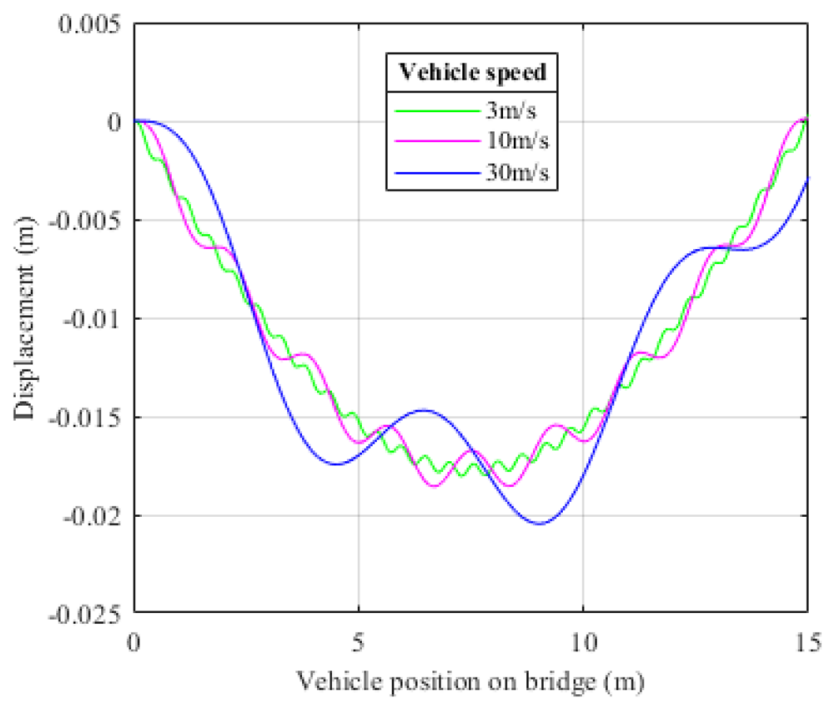

Fig. 2 Bridge midspan displacement response

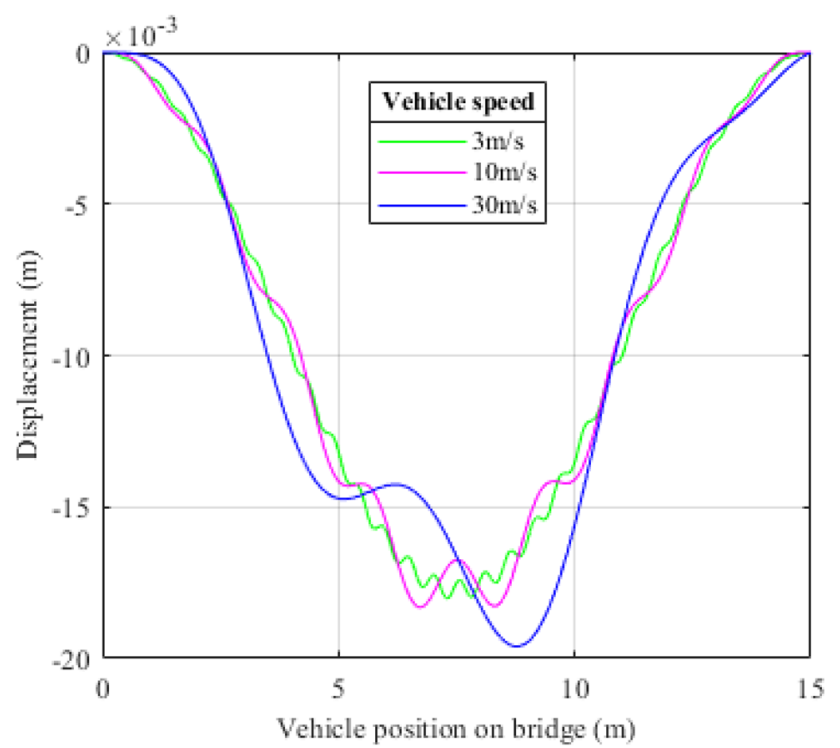

Fig. 3 Bridge total displacement response

Figure 2 and Fig. 3 refer to, respectively, as the bridge midspan and total displacement response to three vehicle's constant speeds, $3 \mathrm{~m} / \mathrm{s}, 10 \mathrm{~m} / \mathrm{s}$, and $30 \mathrm{~m} / \mathrm{s}$. Using different vehicle velocities with the lowest and highest amount has been adopted in previous research, e.g., Bencat [25] and Refs [15, 16], to accurately study the various bridge dynamic behavior. As shown in Fig. 2. and Fig. 3, during the distance that the car is traveling over the bridge, the displacement of the bridge shows the larger scale of oscillation compared to those caused by lower vehicle speeds. For the slowest car speed $(3 \mathrm{~m} / \mathrm{s})$, the scale of fluctuation in the bridge displacement has decreased significantly. Of interest is that the midspan and total deflection curves are almost symmetrical for those caused by vehicle speeds at $3 \mathrm{~m} / \mathrm{s}$ and $10 \mathrm{~m} / \mathrm{s}$. In contrast, for the velocity at $30 \mathrm{~m} / \mathrm{s}$, the corresponding deflection curve is not symmetric.

It is essential to define a static curve originated by the corresponding vehicle during a test and compare its peak value with the bridge's maximum dynamic deflection. The bridge's static response must be obtained via several tests considering a vehicle at a low speed. However, suppose a static test is not performed before a dynamic test. In that case, the bridge displacement caused by the lowest vehicle speed is assumed as the static deflection with sufficient accuracy [25]. For this significant phenomenon, Van Do [26] compared his results obtained via isogeometric and finite element methods to Warburton's analytical results [27]. He noted that a simply supported Bernoulli-Euler bridge is capable to closely approach its static deformation if only the velocity of the vehicle is low enough. Hence it is possible to utilize the static curve obtained by vehicle speed at $3 \mathrm{~m} / \mathrm{s}$ to find the value of dynamic increment, which is represented below [28]:

$\phi=\frac{d_{d y n}-d_{\text {stat }}}{d_{\text {stat }}}$

Table 2 Dynamic increment at central point of the bridge

\begin{tabular}{llll}
\hline $\begin{array}{l}\text { Vehicle speed } \\
(m / s)\end{array}$ & $d_{\text {stat }}(m)$ & $d_{\text {dyn }}(m)$ & $\phi$ \\
\hline 10 & -0.0180 & -0.0186 & 0.0333 \\
30 & -0.0180 & -0.0205 & 0.1389 \\
\hline
\end{tabular}

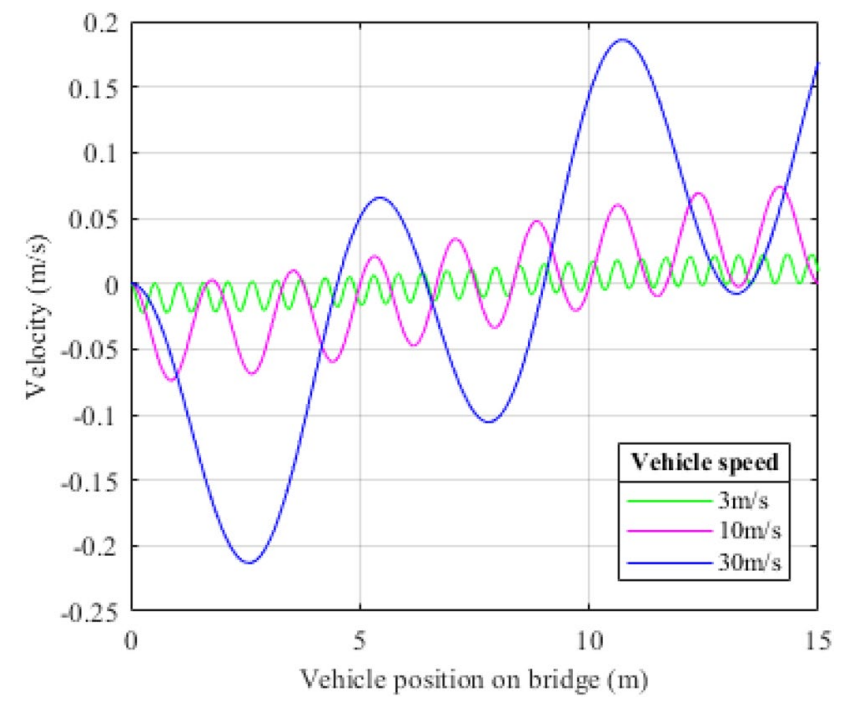

Fig. 4 Bridge midspan velocity response 


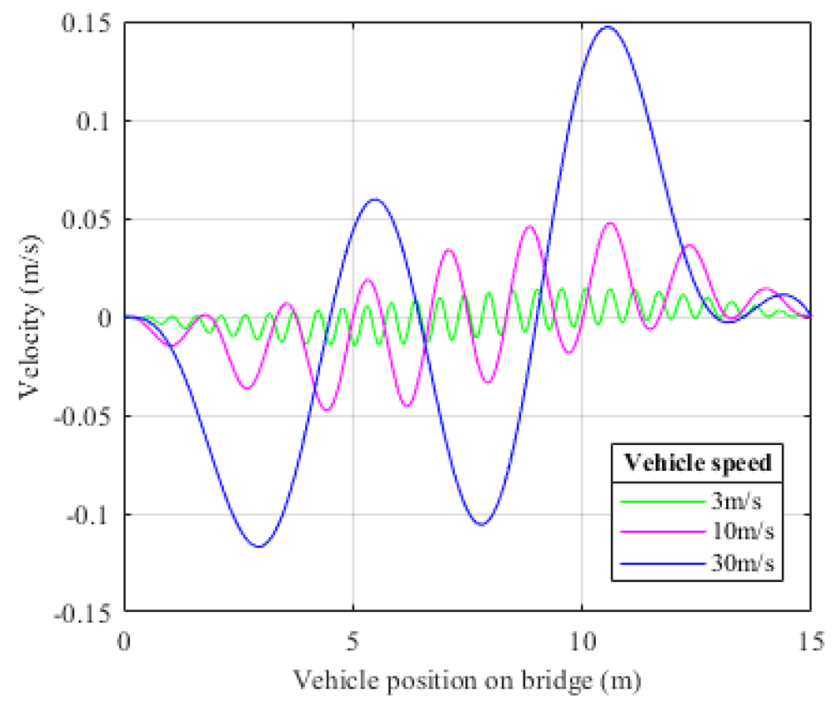

Fig. 5 Bridge total velocity response

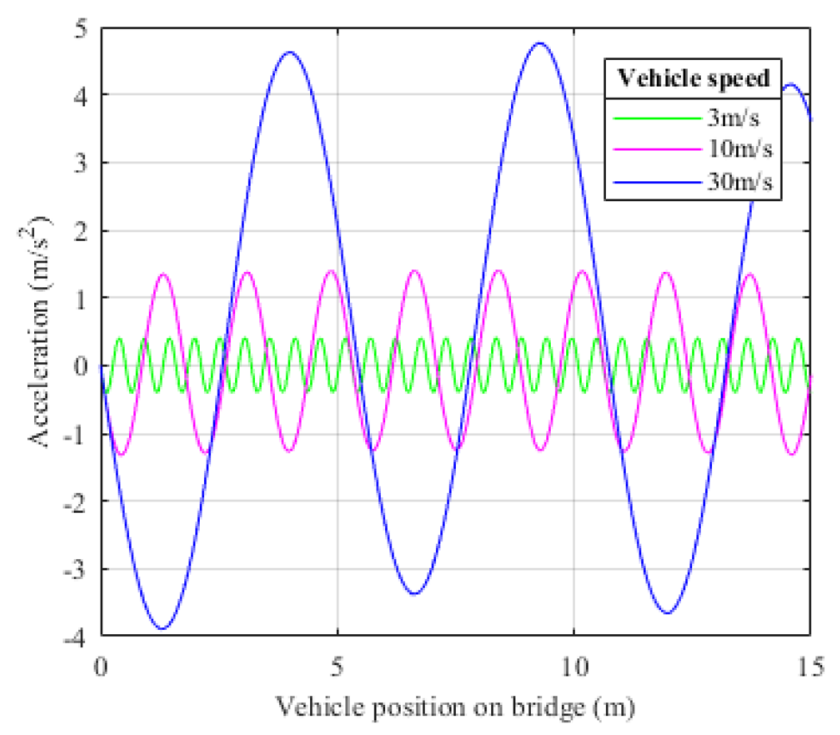

Fig. 6 Bridge midspan Acceleration response

where $d_{d y n}$ is the maximum deflection measured during the vehicle traveling over the bridge and $d_{\text {stat }}$ is the peak value estimated from the static curve.

Table 2 shows the dynamic increment values at the bridge's central point caused by vehicle speeds at 10 and $30 \mathrm{~m} / \mathrm{s}$ and peak values for both static and dynamic deflections caused by the same vehicle. The result shows that as the car's speed triples, the dynamic increment increases by $317.117 \%$. However, it is essential to study the changes in the bridge's dynamic behavior under the influence of environmental conditions such as temperature. Also, the self-weight of a bridge is another parameter that cannot be considered a point mass in theoretical formulas used

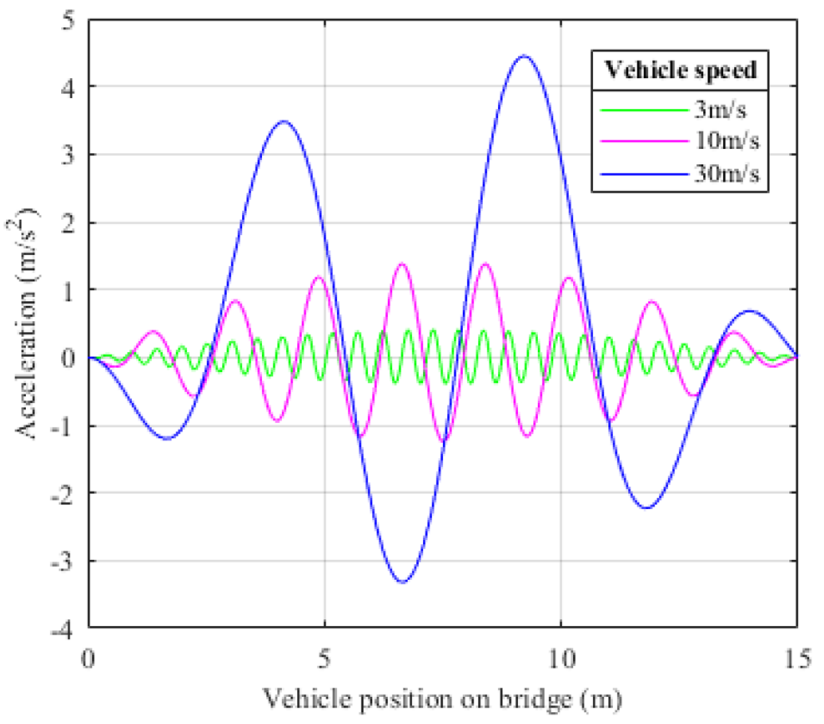

Fig. 7 Bridge total acceleration response

to find the bridge's static deflection. Considering all these notes, the results in Table 2 are just an approximation. It requires practical tests to be carried out to find more accurate values for both static and dynamic deflection for the bridge with previously mentioned properties.

Figures 4, 5, 6 and 7 show the total and central velocity and acceleration responses of the bridge. As can be seen, by applying higher speed to the car, the range of velocity and acceleration of the bridge are increased. As a similar result obtained by Yang [13], the midspan dynamic response of a bridge subjected to a moving 1-DOF quarter car model with linear suspension showed higher acceleration variations when the vehicle's velocity increases. Further, in very recent work done by $\mathrm{Ho}$ and his colleague [29], the effect of traffic flow and the vehicle's speed on a simply supported bridge was studied, assuming the vehicle as a 2-DOF linear model. Using the finite element method (FEM), Ho concluded that the root mean square (RMS) and the bridge's acceleration peak values rise when the traffic flow speed increases. Hence, according to the numerical simulations obtained via MATLAB, it can be concluded that the nonlinear sprung-mass model in this study affects the dynamic bridge behavior as like as linear sprung-mass does. However, practical field tests have to be done for both bridge and vehicle characteristics to prove the study's theoretical approaches.

For a bridge subjected to a moving vehicle with different speed stages, it is significant to measure and compare the total and midspan dynamic responses of the bridge with each other. According to the obtained data, at speed of $3 \mathrm{~m} / \mathrm{s}$, the maximum total and midpoint displacement of the bridge have the same value. However, for speeds at 10 and $30 \mathrm{~m} / \mathrm{s}$ the peak value of midspan deflection 
is, respectively $1.639 \%$ and $4.591 \%$ higher than maximum total displacement. Whereas, the maximum midspan velocity for vehicle speeds at 3,10 and $30 \mathrm{~m} / \mathrm{s}$ is, respectively $53.793 \%, 54.926 \%$ and $-244.935 \%$ higher than peak value of total velocity response of the bridge. On the other hand, the tip value of midpoint acceleration of the bridge for car velocity at 3,10 and $30 \mathrm{~m} / \mathrm{s}$ is, respectively $1.799 \%$, $5.773 \%$ and $7.037 \%$ higher than the maximum value of the total acceleration response. It can conclude that the maximum displacement, velocity, and acceleration happen at the central point of the bridge and has a higher percentage of differences in values compare to total responses at higher vehicle constant speed. Furthermore, by omitting nonlinear stiffness and damping constants from the equations, it was seen that for vehicle speeds at 3,10 and $30 \mathrm{~m} / \mathrm{s}$ neither there was any effect on the acceleration nor the deflection and velocity of the bridge and this was also true for the sprung and unsprung masses of the quarter car model.

\section{Conclusion}

In this study, the dynamic response was obtained using the modal superposition method for the bridge subjected to a moving vehicle. The quarter car model with cubic nonlinearities in a passive suspension system was considered to monitor the bridge's deflection, velocity, and acceleration. Later the dynamic increment of the bridge was measured concerning its approximate static displacement curve. It was seen that by tripling the vehicle speed from 10 to $30 \mathrm{~m} / \mathrm{s}$, the dynamic increment increases. By applying higher speed to the vehicle, it was seen that the displacement of the bridge in both total and midspan cases increases significantly. Plus, it was seen that by neglecting the nonlinear coefficients of the vehicle suspension system, the dynamic response of the bridge remained almost the same for all three velocities which were applied to the car via the numerical calculations. According to the results, the changes in the bridge's dynamic responses are mainly due to the vehicle velocity. The nonlinearity of its suspension system does not affect the bridge deflection in this study. However, it is suggested that field tests are needed to be obtained with more accurate data and validate the theoretical results. Further, it was concluded that the maximum deflection, speed, and acceleration occur at the midpoint of the bridge and has a higher percentage of differences in values compare to total responses at higher car constant velocity. For vehicle speed at $30 \mathrm{~m} / \mathrm{s}$, the maximum amount of bridge midspan displacement, velocity, and acceleration was higher than the peak value of total dynamic responses.
Open Access This article is licensed under a Creative Commons Attribution 4.0 International License, which permits use, sharing, adaptation, distribution and reproduction in any medium or format, as long as you give appropriate credit to the original author(s) and the source, provide a link to the Creative Commons licence, and indicate if changes were made. The images or other third party material in this article are included in the article's Creative Commons licence, unless indicated otherwise in a credit line to the material. If material is not included in the article's Creative Commons licence and your intended use is not permitted by statutory regulation or exceeds the permitted use, you will need to obtain permission directly from the copyright holder. To view a copy of this licence, visit http://creativecommons .org/licenses/by/4.0/.

\section{References}

1. G. B. C. a.t. i. i.t. a. o. i.t. r. structures, Report of the commissioners appointed to inquire into the application of iron to railway structures. Vol. 1123: William Clowes and sons, (1849)

2. Stokes GG (1849) Discussion of a differential equation relating to the breaking of railway bridges. Trans Cambridge Phil Soc 8(5):707-735

3. Peskin L (1935) CE Inglis, a mathematical treatise on vibrations in railway bridges. Bull Am Math Soc 41:315-315

4. Lee $H$ (1996) The dynamic response of a Timoshenko beam subjected to a moving mass. J Sound Vib 198(2):249-256

5. Foda MA, Abduljabbar Z (1998) A dynamic green function formulation for the response of a beam structure to a moving mass. J Sound Vib 210:295-306

6. Mahmoud M, Abou Zaid M (2002) Dynamic response of a beam with a crack subject to a moving mass. J Sound Vibration 256:591-603

7. Azam SE, Mofid M, Khoraskani RA (2013) Dynamic response of Timoshenko beam under moving mass. Sci Iranica 20:50-56

8. Uzzal RUA, Bhat RB, Ahmed W (2012) Dynamic response of a beam subjected to moving load and moving mass supported by Pasternak foundation. Shock Vib 19:201-216

9. Olsson M (1991) On the fundamental moving load problem. J Sound Vib 145:299-307

10. Yang Y-B, Wu C-M, Yau J-D (2001) Dynamic response of a horizontally curved beam subjected to vertical and horizontal moving loads. J Sound Vib 242:519-537

11. Yang Y-B, Yau J-D, Hsu L-C (1997) Vibration of simple beams due to trains moving at high speeds. Eng Struct 19:936-944

12. Yang Y-B, Lin C, Yau J (2004) Extracting bridge frequencies from the dynamic response of a passing vehicle. J Sound Vib 272:471-493

13. Yang $Y$, Lin C (2005) Vehicle-bridge interaction dynamics and potential applications. J Sound Vib 284:205-226

14. Elhattab A, Uddin N, Obrien E (2018) Drive-by bridge frequency identification under operational roadway speeds employing frequency independent underdamped pinning stochastic resonance (FI UPSR),". Sensors 18:4207

15. Goicolea JM, Antolín P (2012) The dynamics of high-speed railway bridges: a review of design issues and new research for lateral dynamics. Int J Railw Technol 1:27-55

16. Deng YJ, Phares BM (2016) Investigation of the effect of speed on the dynamic impact factor for bridges with different entrance conditions. Final Report. InTrans Project 14-521. Institute of Transportation, lowa State University

17. Law S, Zhu X (2004) Dynamic behavior of damaged concrete bridge structures under moving vehicular loads. Eng Struct 26:1279-1293 
18. Deng L, Cai C (2009) Identification of parameters of vehicles moving on bridges. Eng Struct 31:2474-2485

19. Nunia B, Rahman T, Choudhury S, Janardhan P (2020) Effect of vehicle speed and road surface roughness on the impact factor of simply supported bridges due to IRC Class A and B loading. SN Appl Sci 2:1-24

20. Fayyad SM (2012) Constructing control system for active suspension system. Contemp Eng Sci 5:189-200

21. Zhou S, Song G, Wang R, Ren Z, Wen B (2017) Nonlinear dynamic analysis for coupled vehicle -bridge vibration system on nonlinear foundation. Mech Syst Signal Process 87:259-278

22. Yang $Y$, Chang $K$ (2009) Extraction of bridge frequencies from the dynamic response of a passing vehicle enhanced by the EMD technique. J Sound Vib 322:718-739

23. Bao M (2005) Analysis and design principles of MEMS devices. Elsevier, Amsterdam

24. Biggs JM, Biggs JM (1964) Introduction to structural dynamics. McGraw-Hill College, New York
25. Benčat J, Kohár R (2018) Bridges subjected to dynamic loading. Intech Open, Bridge Engineering, Ch. 7, (Edited by Hamid Yaghoubi), p 111-138

26. Van Do VN, Ong TH, Thai CH (2017) Dynamic responses of EulerBernoulli beam subjected to moving vehicles using isogeometric approach. Appl Math Model 51:405-428

27. Warburton GB (2014) The dynamical behaviour of structures: structures and solid body mechanics series. Elsevier, Amsterdam

28. Cantieni R (1983) Dynamic load testing of highway bridges. Transportation Research Record 950, National Academy of Sciences, Washington, D.C., pp 141-148

29. Ho H, Nishio M (2020) Evaluation of dynamic responses of bridges considering traffic flow and surface roughness. Eng Struct 225:111256

Publisher's Note Springer Nature remains neutral with regard to jurisdictional claims in published maps and institutional affiliations. 